Applied and

NISTIR 4321

Computational

Mathematics

Division

Center for Computing and Applied Mathematics

On the Expected Complexity of the 3-Dimensional Voronoi

Diagram

J. Bernal

May 1990 


\section{ON THE EXPECTED COMPLEXITY OF THE 3-DIMENSIONAL VORONOI DIAGRAM}

\section{J. Bernal}

U.S. DEPARTMENT OF COMMERCE Natlonal Institute of Standards and Technolosy

Center for Computing and Applled Mathematlcs

Applled and Computational Mathematlcs Divlsion

Gatthersburg, MD 20899

May 1990

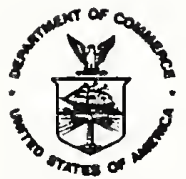

U.S. DEPARTMENT OF COMMERCE Robert A. Mosbacher, Secretary NATIONAL INSTITUTE OF STANDARDS AND TECHNOLOGY

Dr. John W. Lyons, Dlrector 



\title{
ON THE EXPECTED COMPLEXITY OF THE 3-DIMENSIONAL VORONOI DIAGRAM
}

\author{
Javier Bernal \\ Applied and Computational Mathematics Division \\ National Institute of Standards and Technology \\ Gaithersburg, MD 20899
}

Key Words and Phrases: algorithm; computational geometry; expected complexity; expected linear; expected time analysis; Voronoi diagram.

\section{ABSTRACT}

Let $S$ be a set of $n$ sites chosen independently from a uniform distribution in a cube in 3-dimensional Euclidean space. In this paper, work by Bentley, Weide and Yao is extended to show that the Voronoi diagram for $S$ has an expected $O(n)$ number of faces. A consequence of the proof of this result is that the Voronoi diagram for $S$ can be constructed in expected $O(n)$ time.

\section{INTRODUCTION}

Consider a set $S=\left\{p_{1}, \ldots, p_{n}\right\}$ of $n$ sites in $d$-dimensional Euclidean space $E^{d}$. The Voronoi diagram for $S$ is a sequence $V\left(p_{1}\right), \ldots, V\left(p_{n}\right)$ of convex polyhedra covering $E^{d}$, where for each $i, i=1, \ldots, n, V\left(p_{i}\right)$ is the Vorono $i$ polyhedron of $p_{i}$ relative to $S$, i. e. the set of all points $x$ in the space such that $p_{i}$ is as close to $x$ as is any other site in $S$.

The Voronoi diagram is an important geometrical concept that is used for solving a large number of problems in many areas. Accordingly, several algorithms have been devised and implemented for constructing it in two and higher dimensions (see Bentley, Weide and Yao (1980), Bowyer (1981), Brostow, Dussault and Fox (1978), Brown (1979), Finney (1979), Green and Sibson (1978), Lee and Schachter (1980), Maus (1984), Ohya, Iri and Murota (1984), Seidel (1986), Shamos (1978), Shamos and Hoey (1975), Tanemura, Ogawa and Ogita (1983), Watson (1981), Witzgall (1973b)), and many of its statistical and geometrical properties have been derived (see Bentley, et al. (1980), Klee (1980), Lawson (1977), Lee and Schachter (1980), Miles (1970), Paschinger (1982), Preparata (1977), Seidel (1982), Shamos (1978), Shamos and Hoey (1975), Witzgall (1973a)). 
In this paper, we further develop the work by Bentley, Weide and Yao (1980) that relates to the expected complexity of Voronoi diagrams. Given a set $S$ of $n$ sites chosen independently from a uniform distribution in a $d$-dimensional hypercube, Bentley, et al. show that with the exception of at most an expected $O\left(n^{1-1 / d} \cdot \log n\right)$ number of polyhedra, each polyhedron in the Voronoi diagram for $S$ has an expected constant number of faces. With $m$ defined as the largest integer less than or equal to $n^{1 / d}$, i. e. the floor of $n^{1 / d}$, Bentley, et al. first divide the hypercube into $m^{d}$ equal-sized cells. Given $c>0$ and defining $\mathrm{LG}(n)$ as the floor of $c \cdot \log n$, where $\log$ denotes the natural $\operatorname{logarithm}$, Bentley, et al. then show that for each site $p$ in $S$ the expected number of faces of $V(p)$ is constant if $p$ is not constained in any of the outermost LG $(n)$ layers of cells of the hypercube. However, Bentley, et al. leave unclear how to compute the expected complexity of the Voronoi diagram for $S$ due to the Voronoi polyhedra of the sites in the outermost LG $(n)$ layers of cells of the hypercube.

In what follows, we extend the work by Bentley, et al. to show that in 3 -dimensional Euclidean space, $O\left(n^{2 / 3} \cdot(c \cdot \log n)^{4}\right)$ is an upper bound for the expected number of faces of the Voronoi diagram for $S$ that are also faces of Voronoi polyhedra of sites in the outermost LG $(n)$ layers of cells of the cube. This result and those in Bentley, et al. (1980) then imply that the expected number of faces of the Voronoi diagram for the $n$ sites is $O(n)$. Accordingly, we conjecture that in $E^{d}$, for fixed $d>3$, similar results hold for $(d-1)$-dimensional faces or facets, i. e. $O\left(n^{1-1 / d} \cdot(c \cdot \log n)^{d+1}\right)$ is an upper bound for the expected number of facets of the Voronoi diagram for $S$ that are also facets of Voronoi polyhedra of sites in the outermost LG $(n)$ layers of cells of the hypercube, and $O(n)$ is the expected number of facets of the Voronoi diagram for the $n$ sites.

\section{TERMINOLOGY}

Let $S=\left\{p_{1}, \ldots, p_{n}\right\}$ be a set of $n$ points in $E^{3}$ chosen independently from a uniform distribution in a cube $R$. In what follows, a point in $E^{3}$ will be called a site if and only if it belongs to $S$. With $m$ defined as the floor of $n^{1 / 3}$, assume as in Bentley, et al. (1980) that $R$ has been divided into $m^{3}$ equal-sized cells. Given a site $q$, define the $1^{\text {st }}$ layer of cells that surrounds $q$ as the collection of cells that contain $q$. Inductively, given $k \geq 1$, assume that the $k^{\text {th }}$ layer of cells that surrounds $q$ has been defined. Define the $(k+1)^{t h}$ layer of cells that surrounds $q$ as the collection, possibly empty, of cells that have one or more points in common with cells in the $k^{\text {th }}$ layer, and that do not belong to the first $k$ layers.

Let lcell and vcell represent, respectively, the length and volume of each cell. 
Given numbers $c, c^{\prime}, c^{\prime \prime}, 0<c \leq c^{\prime}, c^{\prime \prime} \geq 1$, define $\mathrm{LG}(n)$ and $\mathrm{LG}^{\prime}(n)$ as the floors of $c \cdot \log n$ and $c^{\prime} \cdot \log n$, respectively, and assume $n$ is large enough so that $\mathrm{LG}(n)>2$ and $2^{3 / 2} \cdot c^{\prime \prime} \cdot \mathrm{LG}^{\prime}(n) \leq 2^{-1} \cdot n^{1 / 3}$.

Let $\hat{k}$ denote the largest integer $k$ for which

$$
2^{k / 2} \cdot c^{\prime \prime} \cdot \mathrm{LG}^{\prime}(n) \leq 2^{-1} \cdot n^{1 / 3} .
$$

It follows from the assumptions on $n$ that $\hat{k} \geq 3$.

Set $\mathrm{LG}_{0}(n)$ equal to $\mathrm{LG}(n)$, and $\mathrm{LG}_{k}(n)$ equal to $\mathrm{LG}^{\prime}(n)$ for each $k$, $k=1, \ldots, \hat{k}-2$.

Let $f_{i}, i=1, \ldots, 6$, represent the facets of $R$, and let $\Pi$ denote $\cup_{i=1}^{6} f_{i}$, i. e. the boundary of $R$.

Given a point $x$ in $E^{3}$ and a subset $W$ of $E^{3}$, define $\operatorname{dist}(x, W)$ as the minimum value of $\|x-w\|$ for $w$ in $W$, where $\|\cdot\|$ represents the 3 -dimensional Euclidean norm.

From the assumptions on $n$, several nonempty subsets of $R$ can be defined as follows:

$$
\begin{aligned}
& R_{-1} \equiv\{x \in R: \operatorname{dist}(x, \Pi) \geq \text { lcell } \cdot \mathrm{LG}(n)\} \\
& R_{0} \equiv\{x \in R: \text { lcell } \cdot 2 \leq \operatorname{dist}(x, \Pi)<\text { lcell } \cdot \mathrm{LG}(n)\} \\
& R_{\hat{k}} \equiv\left\{x \in R: \operatorname{dist}(x, \Pi)<\text { lcell } \cdot 2^{-\hat{k}+2}\right\}
\end{aligned}
$$

For each $k, k=1, \ldots, \hat{k}-1$,

$$
R_{k} \equiv\left\{x \in R: \text { lcell } \cdot 2^{-k+1} \leq \operatorname{dist}(x, \Pi)<\text { lcell } \cdot 2^{-k+2}\right\} .
$$

For each $i, k, i=1, \ldots, 6, k=0, \ldots, \hat{k}-2$,

$$
R_{k}^{i} \equiv\left\{x \in R_{k}: \operatorname{dist}\left(x, f_{j}\right) \geq l \text { cell } \cdot 2^{k / 2} \cdot c^{\prime \prime} \cdot \mathrm{LG}_{k}(n), j=1, \ldots, 6, j \neq i\right\} .
$$

It follows from these definitions that the sets $R_{k}, k=-1, \ldots, \hat{k}$, are pair-wise disjoint nested regions of the cube $R$, and

$$
R=\cup_{k=-1}^{\hat{k}} R_{k} \text {. }
$$

Finally, define $R_{-2}$, a possibly empty subset of $R$, as follows:

$$
R_{-2} \equiv\left\{x \in R: \operatorname{dist}(x, \Pi) \geq \text { lcell } \cdot\left(1+c^{\prime \prime}\right) \cdot \operatorname{LG}(n)\right\} .
$$


The significance of these regions as it relates to our purposes can be summarized as follows. $R_{-1}$ is essentially that region of the cube $R$ obtained by subtracting the outermost $L G(n)$ layers of cells of $R$ from $R$. From Bentley, et al. (1980), the Voronoi polyhedron of a site in $R_{-1}$ is of expected constant complexity. $R_{0}$ is essentially that region of $R$ obtained by subtracting from the outermost $\mathrm{LG}(n)$ layers of cells of $R$ the outermost two layers. $R_{k}$, $k=1, \ldots, \hat{k}$, are regions of $R$ whose union is essentially that region of $R$ composed of the outermost two layers of cells of $R$, and whose thicknesses correspond to the terms of the geometric series expanded to the first $\hat{k}-1$ terms together with the remainder. $R_{k}^{i}, i=1, \ldots, 6, k=0, \ldots, \hat{k}-2$, are subsets of $R_{k}, k=0, \ldots, \hat{k}-2$, respectively, defined in such a way that due to their positions relative to the boundary of $R$ and the geometric series aspect of $R_{k}$, $k=1, \ldots, \hat{k}-2$, for a properly selected value of $c^{\prime \prime}$ the expected complexity of the Voronoi diagram for $S$ due to the Voronoi polyhedra of sites in these regions is linear. They are also defined in such a way that due to the definitions of $\hat{k}, R_{\hat{k}-1}$ and $R_{\hat{k}}$, and the geometric series aspect of $R_{k}, k=1, \ldots, \hat{k}$, the expected number of sites in $\cup_{k=0}^{\hat{k}} R_{k} \backslash \cup_{i=1}^{6} \cup_{k=0}^{\hat{k}-2} R_{k}^{i}$ is small enough that it does not affect the linearity of the overall expected complexity of the diagram even under the worst possible circumstances (see Section 3 ). Finally, $R_{-2}$ is a subset of $R_{-1}$ defined in such a way that sites in this region are highly unlikely to have Voronoi neighbors in the outermost $\mathrm{LG}(n)$ layers of cells of $R$ while $R_{-1} \backslash R_{-2}$ is a region of $R$ essentially composed of $O(\mathrm{LG}(n))$ contiguous layers of cells of $R$.

For each facet $f$ of $R$, let $H(f)$ represent the plane that contains $f$, and for each site $q$, let $T^{f}(q)$ represent the point in $f$ that is the perpendicular projection of $q$ onto $f$.

Given $i, k, 1 \leq i \leq 6,0 \leq k \leq \hat{k}-2$, and a site $q$ in $R_{k}^{i}$, let $v, v^{\prime}$ and $v^{\prime \prime}$ be vertices of $R$ in $f_{i}$ for which $v^{\prime}-v$ is perpendicular to $v^{\prime \prime}-v$, and for each $j$, $j=0, \ldots, 8$, define a point $t_{j}$ in $H\left(f_{i}\right)$ by

$$
t_{j} \equiv T^{f_{i}}(q)+\left(v^{\prime}-v\right) \cdot \cos (j \pi / 4)+\left(v^{\prime \prime}-v\right) \cdot \sin (j \pi / 4) .
$$

In addition, for each $j, j=1, \ldots, 8$, let $O_{j}$ be the octant in $H\left(f_{i}\right)$ that is the convex hull of the rays $T^{f_{i}}(q) \vec{t}_{j-1}$ and $T^{f_{i}}(q) \vec{t}_{j}$, and say that $O_{j}, j=1, \ldots, 8$, are the octants associated with $q$. Finally, if within the first $2^{k / 2} \cdot \mathrm{LG}_{k}(n)$ layers of cells that surround $q$, for each $j, j=1, \ldots, 8$, there exists a site $q_{j}$ such that $\operatorname{dist}\left(q_{j}, f_{i}\right)<l$ cell $\cdot 2^{-k}$ and the ray $q \vec{q}_{j}$ intersects $O_{j}$, say that $q$ is octant-closed and that $q_{j}, j=1, \ldots, 8$, render $q$ octant-closed.

Given $i, k, q, v, v^{\prime}, v^{\prime \prime}$ as above, let $v^{\prime \prime \prime}$ be a vertex of $R$ for which $v^{\prime \prime \prime}-v$ is perpendicular to $v^{\prime}-v$ and $v^{\prime \prime}-v$, and for each $j, j=0, \ldots, 8$, and each $m$, 
$m=0, \ldots, 3$, define a point $r_{j m}$ by

$$
\begin{aligned}
r_{j m} \equiv q & +\left(\left(v^{\prime}-v\right) \cdot \cos (j \pi / 4)+\left(v^{\prime \prime}-v\right) \cdot \sin (j \pi / 4)\right) \cdot \sin (m \pi / 4) \\
& +\left(v^{\prime \prime \prime}-v\right) \cdot \cos (m \pi / 4) .
\end{aligned}
$$

In addition, for each $j, j=1, \ldots, 8$, and each $m, m=1,2,3$, let $U_{3 m}$ be the cone that is the convex hull of the rays $q \vec{r}_{j-1, m-1}, q \vec{r}_{j, m-1}, q \vec{r}_{j-1, m}$, and $q \vec{r}_{j m}$, and say that $U_{j m}, j=1, \ldots, 8, m=1,2,3$, are the cones associated with $q$. Finally, if within the first $2^{k / 2} \cdot \mathrm{LG}_{k}(n)$ layers of cells that surround $q$, for each $j, j=1, \ldots, 8$, and each $m, m=1,2,3$, there exists a site $s_{j m}, s_{j m} \neq q$, such that $s_{j m}$ belongs to $U_{j m}$, say that $q$ is cone-closed and that $s_{j m}, j=1, \ldots, 8$, $m=1,2,3$, render $q$ cone-closed.

Given $q$ as above, say that $q$ is closed if it is octant-closed and cone-closed. As it will be shown in Section 3, Voronoi polyhedra of closed sites are of expected complexity acceptable for our purposes.

Given $i, k, q$ as above, define $C^{f_{i}}(q)$ and $C(q)$ as the closed half-spaces that contain $T^{f_{1}}(q)$ and $q$, respectively, and that are determined by the plane parallel to $H\left(f_{i}\right)$ that contains $\left(T^{f_{i}}(q)+q\right) / 2$. Define $S^{f_{i}}(q)$ as the subset of $S$ for which a site $p \in S^{f_{i}}(q)$ if and only if $V(p) \cap V(q) \cap C^{f_{i}}(q) \neq 0$, and $S(q)$ as the subset of $S$ for which a site $p \in S(q)$ if and only if $V(p) \cap V(q) \cap C(q) \neq \emptyset$.

Finally, given sites $p$ and $q$, say that $p$ is a Voronoi neighbor relative to $S$ of $q$ if $V(p)$ and $V(q)$ have a facet in common.

\section{RESULTS}

In this section, based on the terminology developed in Section 2, we prove the following theorem which is the main result of this paper.

Theorem. $O\left(n^{2 / 3} \cdot(c \cdot \log n)^{4}\right)$ is an upper bound for the expected number of faces of the Voronoi diagram for $S$ that are also faces of Voronoi polyhedra of sites in $R \backslash R_{-1}$.

The proof of this theorem consists of partitioning the cube into the regions defined in Section 2 and then computing where necessary the expected number of Voronoi neighbor pairs within and between these regions. It requires some preliminary results which we present in the form of propositions. In the first two propositions it is essentially shown that Voronoi polyhedra of closed sites are of expected complexity acceptable for our purposes. 
Proposition 1. Given $i, k, 1 \leq i \leq 6,0 \leq k \leq \hat{k}-2$, a site $q$ in $R_{k}^{i}$, and octants and sites $O_{j}, q_{j}, j=1, \ldots, 8$, such that $O_{j}, j=1, \ldots, 8$, are the octants associated with $q$, and $q_{j}, j=1, \ldots, 8$, render $q$ octant-closed, if $q^{\prime}$ is a site such that for each $j, j=1, \ldots, 8,\left\|q^{\prime}-q\right\|>\sqrt{2}\left\|q_{j}^{\prime}-q\right\|$, where $q_{j}^{\prime}$ is the intersection of $q \vec{q}_{j}$ and $O_{j}$, then $q^{\prime} \notin S^{f_{i}}(q)$.

Proof. Let $q^{\prime}$ be one such site, and define $J^{\prime}$ as the plane that perpendicularly bisects the line segment $\left[q^{\prime}, q\right]$, and $C^{\prime}$ as the open half-space determined by $J^{\prime}$ that contains $q$. We show that $C^{\prime}$ contains $V(q) \cap C^{f_{i}}(q)$, so that $q^{\prime} \notin S^{f_{i}}(q)$. Assume, without any loss of generality, that $q^{\prime}$ is in $f_{i}, T^{f_{i}}(q) \neq q_{j}^{\prime}$, for each $j, j=1,2, T^{f_{i}}(q) \vec{q}_{1}{ }^{\prime} \neq T^{f_{i}}(q) \vec{q}_{2}{ }^{\prime}$, and $q^{\prime}$ is in the convex hull of $T^{f_{i}}(q) \vec{q}_{1}{ }^{\prime}$ and $T^{f_{1}}(q) \vec{q}_{2}^{\prime}$.

Let $J_{1}^{\prime}$ and $J_{2}^{\prime}$ be the planes that are the perpendicular bisectors of the line segments $\left[q_{1}^{\prime}, q\right]$ and $\left[q_{2}^{\prime}, q\right]$, respectively. Let $B$ be the region that is the intersection of $C^{f_{i}}(q)$ and the closed half-spaces determined by $J_{1}^{\prime}$ and $J_{2}^{\prime}$ that contain $q$. We show $B$ is the convex hull of a region $K^{\prime}$ and a ray $\vec{u}^{\prime}$, both of which lie in $C^{\prime}$. Since $C^{\prime}$ is convex, and $B$ contains $V(q) \cap C^{f_{i}}(q)$, the result then follows.

To this end, let $H^{\prime}$ be the plane that contains $\left(T^{f_{i}}(q)+q\right) / 2$ and is parallel to $H\left(f_{i}\right)$; let $H^{\prime \prime}$ be the plane that contains $q$ and is parallel to $H\left(f_{i}\right)$; let $q^{\prime \prime}, q_{1}^{\prime \prime}$, $q_{2}^{\prime \prime}$ be the perpendicular projections onto $H^{\prime \prime}$ of $q^{\prime}, q_{1}^{\prime}, q_{2}^{\prime}$, respectively; let $h^{\prime}$, $h_{1}^{\prime}, h_{2}^{\prime}$ be the lines that are the intersections of $H^{\prime}$ with $J^{\prime}, J_{1}^{\prime}, J_{2}^{\prime}$, respectively; and let $h^{\prime \prime}, h_{1}^{\prime \prime}, h_{2}^{\prime \prime}$ be the lines in $H^{\prime \prime}$ that perpendicularly bisect $\left[q^{\prime \prime}, q\right],\left[q_{1}^{\prime \prime}, q\right]$, $\left[q_{2}^{\prime \prime}, q\right]$, respectively.

Let $\hat{q}$ be the perpendicular projection of $q$ onto $H^{\prime}$. Define $K^{\prime}$ as the intersection of the half-planes in $H^{\prime}$ determined by $h_{1}^{\prime}$ and $h_{2}^{\prime}$ that contain $\hat{q}$, and $K^{\prime \prime}$ as the intersection of the half-planes in $H^{\prime \prime}$ determined by $h_{1}^{\prime \prime}$ and $h_{2}^{\prime \prime}$ that contain $q$.

In order to show that $C^{\prime} \supseteq K^{\prime}$, we first prove that $\left\|q^{\prime \prime}-q\right\|>\sqrt{2}\left\|q_{j}^{\prime \prime}-q\right\|$ for each $j, j=1,2$. To this end, for each $j, j=1,2$, we have

$$
\begin{aligned}
\left\|q^{\prime \prime}-q\right\|^{2}+\left\|q^{\prime}-q^{\prime \prime}\right\|^{2} & =\left\|q^{\prime}-q\right\|^{2} \\
& >2\left\|q_{j}^{\prime}-q\right\|^{2} \\
& =2\left(\left\|q_{j}^{\prime \prime}-q\right\|^{2}+\left\|q_{j}^{\prime}-q_{j}^{\prime \prime}\right\|^{2}\right) \\
& =2\left\|q_{j}^{\prime \prime}-q\right\|^{2}+2\left\|q_{j}^{\prime}-q_{j}^{\prime \prime}\right\|^{2} .
\end{aligned}
$$

But $\left\|q^{\prime}-q^{\prime \prime}\right\|$ equals $\left\|q_{j}^{\prime}-q_{j}^{\prime \prime}\right\|$ for each $j, j=1,2$, so that

$$
\left\|q^{\prime \prime}-q\right\|^{2}>2\left\|q_{j}^{\prime \prime}-q\right\|^{2}+\left\|q_{j}^{\prime}-q_{j}^{\prime \prime}\right\|^{2}
$$

for each $j, j=1,2$, and the inequalities follow.

Since $q_{1}^{\prime}$ and $q_{2}^{\prime}$ belong to the contiguous octants $O_{1}$ and $O_{2}$, respectively, it 
follows that $h^{\prime \prime}$ does not intersect $K^{\prime \prime}$. But by similar triangles, $h^{\prime \prime}, h_{1}^{\prime \prime}, h_{2}^{\prime \prime}$ are the perpendicular projections onto $H^{\prime \prime}$ of $h^{\prime}, h_{1}^{\prime}, h_{2}^{\prime}$, respectively. Thus, $K^{\prime \prime}$ is the perpendicular projection of $K^{\prime}$ onto $H^{\prime \prime}$, and therefore, $h^{\prime}$ can not intersect $K^{\prime}$, which shows $C^{\prime}$ contains $K^{\prime}$.

In order to obtain $\vec{u}^{\prime}$, let $H^{*}$ be the plane that contains $q, q_{1}^{\prime}$, and $q_{2}^{\prime}$; let $C^{*}$ be the closed half-space determined by $H^{*}$ that contains $T^{f_{i}}(q)$; let $w^{\prime}$ be the line that is the intersection of the planes $J_{1}^{\prime}$ and $J_{2}^{\prime}$; let $J^{*}$ be the plane that contains $q$ and $q^{\prime}$, and that is perpendicular to $H^{*}$; and let $w^{\prime \prime}$ be the perpendicular projection onto $J^{*}$ of $w^{\prime}$.

Since $w^{\prime}$ is perpendicular to $H^{*}$, so is $w^{\prime \prime}$, and since from the definition of $q^{\prime}$, $q^{\prime}$ is not in $C^{*}$, we must have that $w^{\prime \prime}$ contains a ray $\vec{u}^{\prime \prime}$ that lies completely in $C^{\prime} \cap C^{*} \cap C^{f_{2}}(q)$. Therefore, from the definition of $w^{\prime \prime}$, it follows that $w^{\prime}$ must contain a ray $\vec{u}^{\prime}$ that is also contained in $C^{\prime} \cap C^{*} \cap C^{f_{i}}(q)$.

Since $B$ is clearly the convex hull of $K^{\prime}$ and $\vec{u}^{\prime}$, the proof is now complete.

Proposition 2. Given $i, k, 1 \leq i \leq 6,0 \leq k \leq \hat{k}-2$, and a site $q$ in $R_{k}^{i}$, if $q$ is closed then for some constant $M>0$ independent of $q, i, k$ and $n$, the smallest number of contiguous layers of cells that surround $q$ and contain each Voronoi neighbor of $q$ is bounded above by $M \cdot 2^{k / 2} \cdot \mathrm{LG}_{k}(n)$.

Proof. Let $O_{j}, j=1, \ldots, 8$, be octants associated with $q$, let $q_{j}, j=1, \ldots, 8$, be sites that render $q$ octant-closed, and let $s_{j m}, j=1, \ldots, 8, m=1,2,3$, be sites that render $q$ cone-closed.

Using arguments similar to those developed in Bentley, et al. (1980), it can be shown that the existence of the sites $s_{j m}, j=1, \ldots, 8, m=1,2,3$, implies that for some constant $M_{1}>0$ independent of $q, i, k$ and $n$, the smallest number of contiguous layers of cells that surround $q$ and contain $S(q)$ is bounded above by $M_{1} \cdot 2^{k / 2} \cdot \mathrm{LG}_{k}(n)$.

We show a similar result for $S^{f_{i}}(q)$.

For each $j, j=1, \ldots, 8, \operatorname{dist}\left(q_{j}, f_{i}\right)<l c e l l \cdot 2^{-k}$. Thus, by similar triangles, since $q$ is contained in $R_{k}$ so that $\operatorname{dist}\left(q, f_{i}\right) \geq$ lcell $\cdot 2^{-k+1}$, we must have that for each $j, j=1, \ldots, 8,\left\|q_{j}^{\prime}-q\right\| \leq 2\left\|q_{j}-q\right\|$, where $q_{j}^{\prime}$ is the intersection of $q \vec{q}_{j}$ and $O_{j}$.

Thus, if $q^{\prime}$ is a site such that for each $j, j=1, \ldots, 8,\left\|q^{\prime}-q\right\|>2 \sqrt{2}\left\|q_{j}-q\right\|$ then for each $j, j=1, \ldots, 8,\left\|q^{\prime}-q\right\|>\sqrt{2}\left\|q_{j}^{\prime}-q\right\|$, and by Proposition 1 , $q^{\prime} \notin S^{f_{i}}(q)$.

Therefore, since for each $j, j=1, \ldots, 8, q_{j}$ is also contained in the first $2^{k / 2}$. $\mathrm{LG}_{k}(n)$ layers of cells that surround $q$, it follows that for some constant $M_{2}>0$ independent of $q, i, k$ and $n$, the smallest number of contiguous layers of cells that surround $q$ and contain $S^{f_{i}}(q)$ is bounded above by $M_{2} \cdot 2^{k / 2} \cdot \mathrm{LG}_{k}(n)$. The proof of the proposition is now complete since the union of $S(q)$ and $S^{f_{i}}(q)$ contains each Voronoi neighbor of $q$. 
In the next proposition it is shown that the probability that a site is not closed is very small and uniform for all sites to which the definition of a closed site applies.

Proposition 3. Given $i, k, 1 \leq i \leq 6,0 \leq k \leq \hat{k}-2$, and a site $q$ in $R_{k}^{i}$, there exist positive constants $M_{1}$ and $M_{2}$ independent of $q, i, k$ and $n$, such that the probability that $q$ is not closed is bounded above by $M_{1} \cdot \exp \left(-M_{2} \cdot\left(\mathrm{LG}_{k}(n)\right)^{2}\right)$, where exp is the exponential function.

Proof. Let $O_{j}, j=1, \ldots, 8$, be the octants associated with $q$, and let $U_{j m}$, $j=1, \ldots, 8, m=1,2,3$, be the cones associated with $q$.

For each $j, j=1, \ldots, 8$, define $O_{j}^{\prime}$ as the subset of $R$ for which a point $p \in O_{j}^{\prime}$ if and only if $p$ is within the first $2^{k / 2} \cdot \mathrm{LG}_{k}(n)$ layers of cells that surround $q, \operatorname{dist}\left(p, f_{i}\right)<l c e l l \cdot 2^{-k}$, and $q \vec{p}$ intersects $O_{j}$. In addition, for each $j$, $j=1, \ldots, 8$, and each $m, m=1,2,3$, define $U_{j m}^{\prime}$ as the subset of $R$ for which a point $p \in U_{j m}^{\prime}$ if and only if $p$ is within the first $2^{k / 2} \cdot \mathrm{LG}_{k}(n)$ layers of cells that surround $q$, and $p$ is in $U_{j m}$.

From the definition of $R_{k}^{i}$ and since $c^{\prime \prime} \geq 1$, the volume of $\cup_{j=1}^{8} O_{j}^{\prime}$ is then approximately equal to

$$
\begin{aligned}
\left(2 \cdot 2^{k / 2} \cdot \mathrm{LG}_{k}(n)\right)^{2} \cdot\left(2^{-k}\right) \cdot \text { vcell } & =\left(4 \cdot 2^{k} \cdot\left(\mathrm{LG}_{k}(n)\right)^{2}\right) \cdot\left(2^{-k}\right) \cdot \text { vcell } \\
& =4 \cdot\left(\mathrm{LG}_{k}(n)\right)^{2} \cdot \text { vcell },
\end{aligned}
$$

so that for each $j, j=1, \ldots, 8$, the volume of $O_{j}^{\prime}$ is approximately equal to

$$
(1 / 8) \cdot 4 \cdot\left(\mathrm{LG}_{k}(n)\right)^{2} \cdot \text { vcell }=(1 / 2) \cdot\left(\mathrm{LG}_{k}(n)\right)^{2} \cdot \text { vcell } \text {. }
$$

Thus, a positive constant $M_{2}$ exists, independent of $q, i, k$ and $n$, such that for each $j, j=1, \ldots, 8, M_{2} \cdot\left(\mathrm{LG}_{k}(n)\right)^{2} \cdot v$ cell is a lower bound for the volume of $O_{j}^{\prime}$.

Therefore, since for each $j, j=1, \ldots, 8$, each $m, m=1,2,3$, and each $h$, $h=1, \ldots, 8$, the volume of $U_{j m}^{\prime}$ is larger than the volume of $O_{h}^{\prime}$, it follows, using arguments developed in Bentley, et al. (1980), that

$$
(8+8 \cdot 3) \cdot \exp \left(-M_{2} \cdot\left(\mathrm{LG}_{k}(n)\right)^{2}\right)=32 \cdot \exp \left(-M_{2} \cdot\left(\mathrm{LG}_{k}(n)\right)^{2}\right)
$$

is an upper bound for the probability that at least one of $O_{j}^{\prime}, j=1, \ldots, 8$, $U_{j m}^{\prime}, j=1, \ldots, 8, m=1,2,3$, does not contain a site.

Thus, by setting $M_{1}$ equal to 32 , the proof of the proposition is then complete.

In the next four propositions it is shown that due to the positions of $R_{k}^{i}$, $i=1, \ldots, 6, k=0, \ldots, \hat{k}-2$, relative to the boundary of $R$ and the geometric series aspect of $R_{k}, k=1, \ldots, \hat{k}-2$, for a properly selected value of $c^{\prime \prime}$ 
the Voronoi polyhedra of sites in $R_{k}^{i}, i=1, \ldots, 6, k=0, \ldots, \hat{k}-2$, are of acceptable expected complexity. It is also shown that due to the geometric series aspect of $R_{k}, k=1, \ldots, \hat{k}$, and the definitions of $\hat{k}, R_{\hat{k}-1}, R_{\hat{k}}$, and $R_{k}^{i}$, $i=1, \ldots, 6, k=0, \ldots, \hat{k}-2$, in $\cup_{k=0}^{\hat{k}} R_{k} \backslash \cup_{i=1}^{6} \cup_{k=0}^{\hat{k}-2} R_{k}^{i}$ the expected number of sites is small enough to be also acceptable for our purposes.

Proposition 4. Given a site $q$ in $\hat{R} \equiv \cup_{i=1}^{6} R_{0}^{i}$, let $N(q)$ be the number of Voronoi neighbors in $R \backslash R_{-2}$ of $q$. The expected value of $\sum_{q \in \hat{R}} N(q)$ is bounded above by

$$
O\left(n^{2 / 3} \cdot(\operatorname{LG}(n))^{4}\right) \text {. }
$$

Proof. Let $\hat{m}$ be the floor of $n^{1 / 3} \cdot(\operatorname{LG}(n))^{-1}$ and assume $R$ has been divided into $\hat{m}^{3}$ equal-sized cubes. Let $B$ be one such cube and assume $B$ has nonempty intersection with $\hat{R}$.

Let $M$ be as in Proposition 2, and let $B^{\prime}$ be the region in $R$ which is the union of $B$ and the cells in the first $M \cdot \operatorname{LG}(n)$ contiguous layers of cells that surround $B$.

Given $q$ in $B$ let $N^{\prime}(q)$ be the number of Voronoi neighbors in $B^{\prime}$ of $q$.

Let $F$ denote the event that all sites in $B$ are closed.

We define several probabilities and random variables relating to $B$ as follows:

$P_{1} \equiv$ Probability that $F$ occurs.

$P_{2} \equiv$ Probability that $F$ does not occur.

$T \equiv$ Value of $\sum_{q \in B} N(q)$.

$T_{1} \equiv$ Value of $\sum_{q \in B} N(q)$ given that $F$ occurs.

$T_{2} \equiv$ Value of $\sum_{q \in B} N(q)$ given that $F$ does not occur.

$T^{\prime} \equiv$ Value of $\sum_{q \in B} N^{\prime}(q)$.

$T_{1}^{\prime} \equiv$ Value of $\sum_{q \in B} N^{\prime}(q)$ given that $F$ occurs.

$T_{2}^{\prime} \equiv$ Value of $\sum_{q \in B} N^{\prime}(q)$ given that $F$ does not occur.

$W \equiv$ Number of sites in $B$.

$W^{\prime} \equiv$ Number of sites in $B^{\prime}$.

In what follows, given a random variable $X, E(X)$ and $V A R(X)$ denote the expected value and variance of $X$, respectively.

We note that since $W^{\prime}$ is binomially distributed then $V A R\left(W^{\prime}\right)<E\left(W^{\prime}\right)$. Thus,

$$
\begin{aligned}
E\left(T^{\prime}\right) & \leq E\left(W \cdot W^{\prime}\right) \leq E\left(\left(W^{\prime}\right)^{2}\right)=\left(E\left(W^{\prime}\right)\right)^{2}+V A R\left(W^{\prime}\right) \\
& <\left(E\left(W^{\prime}\right)\right)^{2}+E\left(W^{\prime}\right)=O\left(\left((\operatorname{LG}(n))^{3}\right)^{2}\right)+O\left((\operatorname{LG}(n))^{3}\right) \\
& =O\left((\operatorname{LG}(n))^{6}\right) .
\end{aligned}
$$

From Proposition 3, there exist positive constants $M_{1}$ and $M_{2}$ independent of $n$, such that $P_{2} \leq M_{1} \cdot \exp \left(-M_{2} \cdot(\mathrm{LG}(n))^{2}\right) \cdot n$. Thus, $P_{1}$ approaches 1 as $n$ increases, and since $E\left(T^{\prime}\right)=P_{1} \cdot E\left(T_{1}^{\prime}\right)+P_{2} \cdot E\left(T_{2}^{\prime}\right)$, we must have that

$$
E\left(T_{1}^{\prime}\right)=\left(E\left(T^{\prime}\right)-P_{2} \cdot E\left(T_{2}^{\prime}\right)\right) / P_{1}
$$




$$
\begin{aligned}
& \leq E\left(T^{\prime}\right) / P_{1}=O\left((\operatorname{LG}(n))^{6}\right) / P_{1} \\
& =O\left((\operatorname{LG}(n))^{6}\right) .
\end{aligned}
$$

But, from Proposition $2, T_{1} \leq T_{1}^{\prime}$, so that

$$
E\left(T_{1}\right) \leq E\left(T_{1}^{\prime}\right) \leq O\left((\operatorname{LG}(n))^{6}\right) .
$$

Thus,

$$
\begin{aligned}
E(T) & =P_{1} \cdot E\left(T_{1}\right)+P_{2} \cdot E\left(T_{2}\right) \\
& \leq O\left((\mathrm{LG}(n))^{6}\right)+P_{2} \cdot n^{2} \\
& =O\left((\mathrm{LG}(n))^{6}\right) .
\end{aligned}
$$

Finally, since the number of cubes such as $B$ is $O\left(n^{2 / 3} \cdot(\operatorname{LG}(n))^{-2}\right)$, it follows that the expected value of $\sum_{q \in \hat{R}} N(q)$ is bounded above by

$$
O\left(n^{2 / 3} \cdot(\operatorname{LG}(n))^{-2}\right) \cdot O\left((\operatorname{LG}(n))^{6}\right)=O\left(n^{2 / 3} \cdot(\operatorname{LG}(n))^{4}\right),
$$

which completes the proof of the proposition.

Proposition 5. Given $i, k, 1 \leq i \leq 6,1 \leq k \leq \hat{k}-2$, and a site $q$ in $R_{k}^{i}$, let $N(q)$ be the number of Voronoi neighbors in $\cup_{l=0}^{\hat{k}} R_{l} \backslash \cup_{l=0}^{k-1} R_{l}^{i}$ of $q$. For constants $M, M^{\prime}>0$ independent of $i, k$ and $n$, if $c^{\prime \prime} \geq(2+\sqrt{2}) \cdot M$ then the expected value of $\sum_{q \in R_{k}^{i}} N(q)$ is bounded above by

$$
M^{\prime} \cdot 2^{-k} \cdot n^{2 / 3} \cdot\left(\mathrm{LG}^{\prime}(n)\right)^{2} .
$$

Proof. Define $\hat{R}_{k}^{i}$, a nonempty subset of $R$ that contains $R_{k}^{i}$, as follows:

$$
\hat{R}_{k}^{i} \equiv\left\{x \in R: \text { lcell } \cdot 2^{-k+1} \leq \operatorname{dist}\left(x, f_{i}\right)<\text { lcell } \cdot 2^{-k+2}\right\} .
$$

Let $\hat{m}$ be the floor of $n^{1 / 3} \cdot\left(2^{k / 2} \cdot \mathrm{LG}^{\prime}(n)\right)^{-1}$ and assume $\hat{R}_{k}^{i}$ has been divided into $\hat{m}^{2}$ equal-sized slabs of thickness $2^{-k+1}$. Let $\dot{B}$ be one such slab and assume $B \equiv \tilde{B} \cap R_{k}^{i}$ is not empty.

Let $M$ be as in Proposition 2, let $\hat{B}$ be the union of the cells in the first $M \cdot 2^{k / 2} \cdot \mathrm{LG}^{\prime}(n)$ contiguous layers of cells that surround $B$, and let $B^{\prime}$ be the region in $R$ which is the union of $B$ and $\hat{B} \cap\left(\cup_{l=0}^{\hat{k}} R_{l} \backslash \cup_{l=0}^{k-1} R_{l}^{i}\right)$.

We compute the expected number of sites in $B^{\prime}$.

$c^{\prime \prime} \geq(2+\sqrt{2}) \cdot M$ and $c^{\prime} \geq c$ imply that

$$
c^{\prime \prime} \cdot 2^{k / 2} \cdot \mathrm{LG}^{\prime}(n)-M \cdot 2^{k / 2} \cdot \mathrm{LG}^{\prime}(n) \geq c^{\prime \prime} \cdot 2^{(k-1) / 2} \cdot \mathrm{LG}_{k-1}(n),
$$

so that $B^{\prime}$ is contained in $\cup_{l=k}^{\hat{k}} R_{l}$ and therefore, for some constant $M^{\prime \prime}>0$ independent of $i, k$ and $n$, has a volume bounded above by

$$
\begin{aligned}
& \left(M^{\prime \prime} \cdot 2^{k / 2} \cdot \mathrm{LG}^{\prime}(n)\right)^{2} \cdot\left(\left(\sum_{l=k}^{\hat{k}-1} 2^{-l+1}\right)+2^{-\hat{k}+2}\right) \cdot \text { vcell } \\
= & \left(M^{\prime \prime}\right)^{2} \cdot 2^{k} \cdot\left(\mathrm{LG}^{\prime}(n)\right)^{2} \cdot\left(2^{-k+2}\right) \cdot \text { vcell } \\
= & 4 \cdot\left(M^{\prime \prime}\right)^{2} \cdot\left(\mathrm{LG}^{\prime}(n)\right)^{2} \cdot \text { vcell. }
\end{aligned}
$$


Thus, $4 \cdot\left(M^{\prime \prime}\right)^{2} \cdot\left(\mathrm{LG}^{\prime}(n)\right)^{2}$ is an upper bound for the expected number of sites in $B^{\prime}$.

Using arguments similar to those employed in the proof of Proposition 4, we can now show that for some constant $M^{\prime}>0$ independent of $i, k$ and $n$, the expected value of $\sum_{q \in B} N(q)$ is bounded above by $M^{\prime} \cdot\left(\operatorname{LG}^{\prime}(n)\right)^{4}$. Therefore, since $n^{2 / 3} \cdot\left(2^{k / 2} \cdot \mathrm{LG}^{\prime}(n)\right)^{-2}$ is an upper bound for the number of slabs such as $\bar{B}$, it follows that the expected value of $\sum_{q \in R_{k}^{i}} N(q)$ is bounded above by

$$
n^{2 / 3} \cdot\left(2^{k / 2} \cdot \mathrm{LG}^{\prime}(n)\right)^{-2} \cdot M^{\prime} \cdot\left(\mathrm{LG}^{\prime}(n)\right)^{4}=M^{\prime} \cdot 2^{-k} \cdot n^{2 / 3} \cdot\left(\mathrm{LG}^{\prime}(n)\right)^{2},
$$

which completes the proof of the proposition.

Proposition 6. The expected number of sites in $\cup_{l=\hat{k}-1}^{\hat{k}} R_{l}$ is bounded above by

$$
384 \cdot\left(c^{\prime \prime} \cdot \mathrm{LG}^{\prime}(n)\right)^{2} .
$$

Proof. Since $\hat{k}$ is the largest integer $k^{\prime}$ for which

$$
2^{k^{\prime} / 2} \cdot c^{\prime \prime} \cdot \mathrm{LG}^{\prime}(n) \leq 2^{-1} \cdot n^{1 / 3}
$$

we must have that the volume of $\cup_{l=\hat{k}-1}^{\hat{k}} R_{l}$ is bounded above by

$$
\begin{aligned}
& 6 \cdot\left(2 \cdot 2^{(\hat{k}+1) / 2} \cdot c^{\prime \prime} \cdot \mathrm{LG}^{\prime}(n)\right)^{2} \cdot\left(2^{-\hat{k}+2}+2^{-\hat{k}+2}\right) \cdot \text { vcell } \\
= & 6 \cdot\left(4 \cdot 2^{\hat{k}+1} \cdot\left(c^{\prime \prime} \cdot \mathrm{LG}^{\prime}(n)\right)^{2}\right) \cdot\left(2 \cdot 2^{-\hat{k}+2}\right) \cdot \text { vcell } \\
= & 384 \cdot\left(c^{\prime \prime} \cdot \mathrm{LG}^{\prime}(n)\right)^{2} \cdot \text { vcell, }
\end{aligned}
$$

which completes the proof of the proposition.

Proposition 7. The expected number of sites in $R_{0} \backslash \cup_{i=1}^{6} R_{0}^{i}$ is bounded above by

$$
12 \cdot n^{1 / 3} \cdot\left(c^{\prime \prime} \cdot \operatorname{LG}(n)\right)^{2},
$$

and in $\cup_{l=1}^{\hat{k}-2} R_{l} \backslash \cup_{i=1}^{6}\left(\cup_{l=1}^{\hat{k}-2} R_{l}^{i}\right)$ by

$$
(1+\sqrt{2}) \cdot 48 \cdot n^{1 / 3} \cdot c^{\prime \prime} \cdot \mathrm{LG}^{\prime}(n) .
$$

Proof. From the definitions, the volume of $R_{0} \backslash \cup_{i=1}^{6} R_{0}^{i}$ is bounded above by

$$
12 \cdot n^{1 / 3} \cdot\left(c^{\prime \prime} \cdot \mathrm{LG}(n)\right)^{2} \cdot \text { vcell, }
$$

and that of $\cup_{l=1}^{\hat{k}-2} R_{l} \backslash \cup_{i=1}^{6}\left(\cup_{l=1}^{\hat{k}-2} R_{l}^{i}\right)$ by

$$
\begin{aligned}
& \sum_{l=1}^{\hat{k}-2} 2 \cdot 12 \cdot n^{1 / 3} \cdot 2^{l / 2} \cdot c^{\prime \prime} \cdot \mathrm{LG}^{\prime}(n) \cdot 2^{-l+1} \cdot \text { vcell } \\
= & \sum_{l=1}^{\hat{k}-2} 48 \cdot n^{1 / 3} \cdot 2^{-l / 2} \cdot c^{\prime \prime} \cdot \mathrm{LG}^{\prime}(n) \cdot \text { vcell } \\
\leq & (1+\sqrt{2}) \cdot 48 \cdot n^{1 / 3} \cdot c^{\prime \prime} \cdot \mathrm{LG}^{\prime}(n) \cdot \text { vcell. }
\end{aligned}
$$


The proposition now follows.

Proof of the theorem. It suffices to prove the theorem for the 2-dimensional faces or facets, since from Klee (1966) and the Euler formula the number of vertices and edges of a 3-dimensional polyhedron is bounded above by a constant times the number of facets of the polyhedron. As mentioned above, the proof consists of computing where necessary the expected number of Voronoi neighbor pairs within and between the regions $R_{k}^{i}, i=1, \ldots, 6, k=0, \ldots, \hat{k}-2$, $\cup_{k=0}^{\hat{k}} R_{k} \backslash \cup_{i=1}^{6} \cup_{k=0}^{\hat{k}-2} R_{k}^{i}, R_{-1} \backslash R_{-2}$ and $R_{-2}$.

To this end, let $p$ be a site in $R_{-1}$.

Since for each site $q$ in $\cup_{k=1}^{\hat{k}} R_{k}$, dist $\left(q, R_{-1}\right) \geq$ lcell $\cdot(\mathrm{LG}(n)-2)$, from Bentley, et al. (1980) we must have that constants $M_{1}^{\prime}$ and $M_{2}^{\prime}>0$ exist independent of $n$ and $p$, such that the probability that $p$ has Voronoi neighbors in $\cup_{k=1}^{k} R_{k}$ is bounded above by

$$
M_{1}^{\prime} \cdot \exp \left(-M_{2}^{\prime} \cdot(\mathrm{LG}(n))^{3}\right) .
$$

Therefore, the expected number of facets of the Voronoi diagram for $S$ that are shared by Voronoi polyhedra of sites in $R_{-1}$ with Voronoi polyhedra of sites in $\cup_{k=1}^{\hat{k}} R_{k}$ is bounded above by

$$
n \cdot n \cdot M_{1}^{\prime} \cdot \exp \left(-M_{2}^{\prime} \cdot(\operatorname{LG}(n))^{3}\right) .
$$

Similarly, positive constants $M_{1}^{\prime \prime}$ and $M_{2}^{\prime \prime}$ exist independent of $n$, such that the expected number of facets of the Voronoi diagram for $S$ that are shared by Voronoi polyhedra of sites in $R_{-2}$ with Voronoi polyhedra of sites in $R_{0}$ is bounded above by

$$
n \cdot n \cdot M_{1}^{\prime \prime} \cdot \exp \left(-M_{2}^{\prime \prime} \cdot(\mathrm{LG}(n))^{3}\right)
$$

For each $i, i=1, \ldots, 6$, let $R_{-1}^{i}$ denote the possibly empty subset of $R_{-1} \backslash R_{-2}$

$$
\left\{x \in R_{-1} \backslash R_{-2}: \operatorname{dist}\left(x, f_{j}\right) \geq \text { lcell } \cdot\left(1+c^{\prime \prime}\right) \cdot \operatorname{LG}(n), j=1, \ldots, 6, j \neq i\right\} .
$$

Given $i, 1 \leq i \leq 6$, let $p$ be a site in $R_{-1}^{i}$.

Since for each site $q$ in $R_{0} \backslash \cup_{j=1}^{\ell} R_{0}^{j}, \operatorname{dist}\left(q, R_{-1}^{i}\right) \geq$ lcell $\cdot \operatorname{LG}(n)$, from Bentley, et al. (1980) we must have again that constants $M_{1}^{\prime \prime \prime}$ and $\boldsymbol{M}_{2}^{\prime \prime \prime}>0$ exist independent of $i, n$ and $p$, such that the probability that $p$ has Voronoi neighbors in $R_{0} \backslash \cup_{j=1}^{6} R_{0}^{j}$ is bounded above by

$$
M_{1}^{\prime \prime \prime} \cdot \exp \left(-M_{2}^{\prime \prime \prime} \cdot(\operatorname{LG}(n))^{3}\right) .
$$

Also, as in the proof of Proposition 7, it follows that the expected number of sites in $\left(R_{-1} \backslash R_{-2}\right) \backslash \cup_{j=1}^{6} R_{-1}^{j}$ is bounded above by $12 \cdot n^{1 / 3} \cdot\left(c^{\prime \prime} \cdot \operatorname{LG}(n)\right)^{2}$. Thus, since the number of sites in $\left(R_{0} \backslash \cup_{j=1}^{6} R_{0}^{j}\right) \cup\left(\left(R_{-1} \backslash R_{-2}\right) \backslash \cup_{j=1}^{6} R_{-1}^{j}\right)$ is 
binomially distributed so that its variance is less than its expected value, it must follow from Proposition 7 that the expected number of facets of the Voronoi diagram for $S$ that are shared by Voronoi polyhedra of sites in $R_{-1} \backslash R_{-2}$ with Voronoi polyhedra of sites in $R_{0} \backslash \cup_{j=1}^{6} R_{0}^{j}$ is bounded above by

$$
\begin{aligned}
& n \cdot n \cdot M_{1}^{\prime \prime \prime} \cdot \exp \left(-M_{2}^{\prime \prime \prime} \cdot(\operatorname{LG}(n))^{3}\right)+ \\
& \left(24 \cdot n^{1 / 3} \cdot\left(c^{\prime \prime} \cdot \operatorname{LG}(n)\right)^{2}\right)^{2}+24 \cdot n^{1 / 3} \cdot\left(c^{\prime \prime} \cdot \operatorname{LG}(n)\right)^{2} .
\end{aligned}
$$

Therefore, since the number of sites in $\cup_{l=0}^{\hat{k}} R_{l} \backslash \cup_{i=1}^{6} \cup_{l=0}^{\hat{k}-2} R_{l}^{i}$ is also binomially distributed so that its variance is also less than its expected value, it must follow from Propositions 4, 5,6 and 7 that constants $M^{\prime}$ and $c^{\prime \prime}>0$ exist independent of $n$ such that the expected number of facets of the Voronoi diagram for $S$ that are also facets of Voronoi polyhedra of sites in $R \backslash R_{-1}$ is bounded above by

$$
\begin{aligned}
& O\left(n^{2 / 3} \cdot(\mathrm{LG}(n))^{4}\right)+6 \cdot \sum_{k=1}^{k-2} M^{\prime} \cdot 2^{-k} \cdot n^{2 / 3} \cdot\left(\mathrm{LG}^{\prime}(n)\right)^{2}+ \\
& \left(384 \cdot\left(c^{\prime \prime} \cdot \mathrm{LG}^{\prime}(n)\right)^{2}+12 \cdot n^{1 / 3} \cdot\left(c^{\prime \prime} \cdot \mathrm{LG}(n)\right)^{2}+\right. \\
& \left.(1+\sqrt{2}) \cdot 48 \cdot n^{1 / 3} \cdot c^{\prime \prime} \cdot \mathrm{LG}^{\prime}(n)\right)^{2}+ \\
& 384 \cdot\left(c^{\prime \prime} \cdot \mathrm{LG}^{\prime}(n)\right)^{2}+12 \cdot n^{1 / 3} \cdot\left(c^{\prime \prime} \cdot \mathrm{LG}(n)\right)^{2}+ \\
& (1+\sqrt{2}) \cdot 48 \cdot n^{1 / 3} \cdot c^{\prime \prime} \cdot \mathrm{LG}^{\prime}(n)+ \\
& n^{2} \cdot M_{1}^{\prime} \cdot \exp \left(-M_{2}^{\prime} \cdot(\mathrm{LG}(n))^{3}\right)+ \\
& n^{2} \cdot M_{1}^{\prime \prime} \cdot \exp \left(-M_{2}^{\prime \prime} \cdot(\mathrm{LG}(n))^{3}\right)+ \\
& n^{2} \cdot M_{1}^{\prime \prime \prime} \cdot \exp \left(-M_{2}^{\prime \prime \prime} \cdot(\mathrm{LG}(n))^{3}\right)+ \\
& \left(24 \cdot n^{1 / 3} \cdot\left(c^{\prime \prime} \cdot \mathrm{LG}(n)\right)^{2}\right)^{2}+24 \cdot n^{1 / 3} \cdot\left(c^{\prime \prime} \cdot \mathrm{LG}(n)\right)^{2} \\
= & M \cdot n^{2 / 3} \cdot(\mathrm{LG}(n))^{4} \\
= & M \cdot n^{2 / 3} \cdot(c \cdot \log n)^{4},
\end{aligned}
$$

where $M$ is a function of $n, c$ and $c^{\prime}$ that decreases for fixed $c$ and $c^{\prime}, 0<c \leq c^{\prime}$. This completes the proof of the theorem.

The following corollary is a direct consequence of results in Bentley, et al. (1980) and the theorem.

Corollary. $O(n)$ is the expected number of faces of the Voronoi diagram for $S$.

Proof. From Bentley, et al. (1980) there exist positive constants $M_{1}^{\prime}$ and $M_{2}^{\prime}$ independent of $n$ such that

$$
O(1)+n \cdot M_{1}^{\prime} \cdot \exp \left(-M_{2}^{\prime} \cdot(\operatorname{LG}(n))^{3}\right)
$$


is the expected number of faces of the Voronoi diagram for $S$ that are also faces of the Voronoi polyhedron of any given site in $R_{-1}$. Thus, from the theorem, the expected number of faces of the Voronoi diagram for $S$ is

$$
n \cdot\left(O(1)+n \cdot M_{1}^{\prime} \cdot \exp \left(-M_{2}^{\prime} \cdot(c \cdot \log n)^{3}\right)+O\left(n^{2 / 3} \cdot(c \cdot \log n)^{4}\right)=M \cdot n,\right.
$$

where $M$ is a function of $n, c$ and $c^{\prime}$ that decreases for fixed $c$ and $c^{\prime}, 0<c \leq c^{\prime}$.

The geometrical nature of the proofs of the theorem and the corollary, and the fact that $O\left(n^{2}\right)$ is the maximum number of facets that the Voronoi diagram for a set of $n$ sites in $E^{d}, d \geq 3$, can have (see Klee (1980), Paschinger (1982), Preparata (1977), Seidel (1982)), suggest the following conjecture. Here, it is assumed that $S$ is a set of $n$ sites in $E^{d}, d>3$, chosen independently from a uniform distribution in a $d$-dimensional hypercube $R$, and that $R$ has been divided into $m^{d}$ equal-sized cells, where $m$ is the floor of $n^{1 / d}$.

Conjecture. For fixed $d, O\left(n^{1-1 / d} \cdot(c \cdot \log n)^{d+1}\right)$ is an upper bound for the expected number of facets or $(d-1)$-dimensional faces of the Voronoi diagram for $S$ that are also facets of Voronoi polyhedra of sites in the outermost LG $(n)$ layers of cells of $R$. Consequently, $O(n)$ is the expected number of facets of the Voronoi diagram for $S$.

The following remark relates to the expected number of faces of the convex hull of $S$.

Remark. From Bentley, et al. (1980) there exist positive constants $M_{1}^{\prime}$ and $M_{2}^{\prime}$ independent of $n$ such that the probability that the Voronoi polyhedron of any site in $R_{-1}$ is unbounded is bounded above by

$$
M_{1}^{\prime} \cdot \exp \left(-M_{2}^{\prime} \cdot(\operatorname{LG}(n))^{3}\right) .
$$

From Proposition 3 and the definition of a closed site there exist positive constants $M_{1}$ and $M_{2}$ independent of $n$ such that the probability that the Voronoi polyhedron of any site in $\cup_{i=1}^{6} \cup_{k=0}^{\hat{k}-2} R_{k}^{i}$ is unbounded is bounded above by

$$
M_{1} \cdot \exp \left(-M_{2} \cdot(\operatorname{LG}(n))^{2}\right) .
$$

Thus, from Propositions 6 and 7, Klee (1966) and the Euler formula, the expected number of faces of the convex hull of $S$ is bounded above by

$$
\begin{aligned}
& n \cdot M_{1}^{\prime} \cdot \exp \left(-M_{2}^{\prime} \cdot(\mathrm{LG}(n))^{3}\right)+n \cdot M_{1} \cdot \exp \left(-M_{2} \cdot(\mathrm{LG}(n))^{2}\right)+ \\
& 384 \cdot\left(c^{\prime \prime} \cdot \mathrm{LG}^{\prime}(n)\right)^{2}+12 \cdot n^{1 / 3} \cdot\left(c^{\prime \prime} \cdot \mathrm{LG}(n)\right)^{2}+ \\
& (1+\sqrt{2}) \cdot 48 \cdot n^{1 / 3} \cdot c^{\prime \prime} \cdot \mathrm{LG}^{\prime}(n) \\
= & O\left(n^{1 / 3} \cdot(\log n)^{2}\right) .
\end{aligned}
$$




\section{SUMMARY}

Let $S$ be a set of $n$ sites chosen independently from a uniform distribution in a $d$-dimensional hypercube $R$, and assume $R$ has been divided into $m^{d}$ equal-sized cells, where $m$ is the floor of $n^{1 / d}$. In addition, let $c$ and $c^{\prime}$ be positive numbers, and define $\mathrm{LG}(n)$ as the floor of $c \cdot \log n$, where $\log$ denotes the natural logarithm. Influenced by Bentley, Weide and Yao's work (1980), we have shown that if $d$ equals 3 then $M \cdot n^{1-1 / d} \cdot(c \cdot \log n)^{d+1}$ is an upper bound for the expected number of facets of the Voronoi diagram for $S$ that are also facets of Voronoi polyhedra of sites in the outermost LG $(n)$ layers of cells of $R$, where $M$ is a function of $n, c$ and $c^{\prime}$ that decreases for fixed $c$ and $c^{\prime}$, $0<c \leq c^{\prime}$. Subsequently, from this result and results in Bentley, et al. (1980), we have shown that $O(n)$ is an upper bound for the expected number of facets of the Voronoi diagram for $S$. Accordingly, we have conjectured that similar results hold for fixed $d>3$, and from Klee (1966) and the Euler formula have concluded that for $d=3$, the same results hold for the 0 - and 1 -dimensional faces of the Voronoi diagram for $S$.

Actually, without explicitly stating it, we have established the existence of an expected $O(n)$ algorithm for constructing Voronoi diagrams in three dimensions. To see this, we note that for each site in the outermost LG $(n)$ layers of cells of $R$, we have implicitly shown the feasibility of obtaining a subset of $S$ that contains all of the Voronoi neighbors of the site. This is done in such a way that the expected time involved in obtaining all such subsets for all such sites is bounded above by $M \cdot n^{2 / 3} \cdot(c \cdot \log n)^{4}$, where $M$ is a function of $n, c$ and $c^{\prime}$ that decreases for fixed $c$ and $c^{\prime}, 0<c \leq c^{\prime}$. Thus, since the intersection of $k$ half-spaces in 3-dimensional space can be found in time $O(k \cdot \log k)$ (see Preparata and Muller (1979)), a computation can be carried out to show that the Voronoi polyhedra of the sites in the outermost LG $(n)$ layers of $R$ can be found in at most

$$
O\left(n^{2 / 3} \cdot(c \cdot \log n)^{4}\right) \cdot \log n=M^{\prime} \cdot n^{2 / 3} \cdot(c \cdot \log n)^{4} \cdot \log n
$$

expected time, where $M^{\prime}$ is a function of $n, c$ and $c^{\prime}$ that decreases for fixed $c$ and $c^{\prime}, 0<c \leq c^{\prime}$. This observation, together with results in Bentley, et al. (1980), then shows the existence of the algorithm. We note that an implementation of this algorithm is currently being tested at the National Institute of Standards and Technology, and computational results obtained from our tests will be presented in a forthcoming paper. It should also be noted that the techniques employed in this algorithm are quite different from those used in an expected $O(n)$ algorithm recently presented in Dwyer (1988) for constructing Voronoi diagrams for point sets chosen uniformly from the $d$-dimensional ball. 


\section{ACKNOWLEDGMENTS}

The author wishes to express his gratitude to Jim Lawrence and Christoph Witzgall for their criticism and suggestions on the paper.

\section{BIBLIOGRAPHY}

Bentley J. L., Weide B. W. and Yao A. C. (1980), Optimal expected-time algorithms for closest point problems, ACM Trans. Math. Software 6, No. 4, 563-580.

Bowyer A. (1981), Computing Dirichlet tessellations, Comput. J. 24, No. 2, 162-166.

Brostow W., Dussault J-P. and Fox B. L. (1978), Construction of Voronoi polyhedra, J. Comput. Phys. 29, 81-92.

Brown K. Q. (1979), Voronoi diagrams from convex hulls, Inform. Process. Lett. 9, No. 5, 223-228.

Dwyer R. A. (1988), Higher-dimensional Voronoi diagrams in linear expected time, Tech. Rep. CMU-CS-88-100, Dept. of Comput. Science, CarnegieMellon Univ.

Finney J. L. (1979), A procedure for the construction of Voronoi polyhedra, J. Comput. Phys. 32, 137-143.

Green P. J. and Sibson R. (1978), Computing Dirichlet tessellations in the plane, Comput. J. 21, No. 2, 168-173.

Klee V. (1966), Convex polytopes and linear programming, Proc. IBM Sci. Comput. Symp. Combinatorial Problems, IBM, White Plains, NY, 123-158.

Klee V. (1980), On the complexity of $d$-dimensional Voronoi diagrams, Arch. Math. 34, 75-80.

Lawson C. L. (1977), Software for $C^{1}$ surface interpolation, in Mathematical Software III (J. R. Rice, Ed.), pp. 161-194, Academic Press, New York.

Lee D. T. and Schachter B. J. (1980), Two algorithms for constructing a Delaunay triangulation, Internat. J. Comput. Inform. Sci. 9, No. 3, 219-242.

Maus A. (1984), Delaunay triangulation and the convex hull of $n$ points in 
expected linear time, BIT 24, 151-163.

Miles R. E. (1970), On the homogeneous planar Poisson point process, Math. Biosci. 6, 85-127.

Ohya T., Iri M. and Murota K. (1984), A fast Voronoi-diagram algorithm with quaternary tree bucketing, Inform. Process. Lett. 18, No. 4, 227-231.

Paschinger I. (1982), Konvexe Polytope und Dirichletsche Zellenkomplexe, Ph. D. dissertation, Math. Inst., Univ. Salzburg.

Preparata F. P. (1977), Steps into computational geometry, Tech. Rep., Coordinated Science Laboratory, University of Illinois.

Preparata F. P. and Muller D. E. (1979), Finding the intersection of $n$ halfspaces in time $O(n \log n)$, Theoret. Comput. Sci. 8, 45-55.

Seidel R. (1982), The complexity of Voronoi diagrams in higher dimensions, in Proc. of the $20^{\text {th }}$ Allerton Conference on Communication, Control, and Computing.

Seidel R. (1986), Constructing higher-dimensional convex hulls at logarithmic cost per face, in Proc. $18^{\text {th }}$ ACM Sympos. on Theory of Computing.

Shamos M. I. (1978), Computational geometry, Ph.D. dissertation, Yale University.

Shamos M. I. and Hoey D. (1975), Closest-point problems, in Proc. $16^{\text {th }}$ Ann. IEEE Symp. on the Foundations of Comp. Sci., pp. 151-162.

Tanemura M., Ogawa T. and Ogita N. (1983), A new algorithm for threedimensional Voronoi tessellation, J. Comput. Phys. 51, 191-207.

Watson D. F. (1981), Computing the $n$-dimensional Delaunay tessellation with application to Voronoi polytopes, Comput. J. 24, No. 2, 167-172.

Witzgall C. J. (1973a), Euclidean neighbor relations, Boeing Document D180-15462-1, Boeing Aerospace Company, Seattle, Washington.

Witzgall C. J. (1973b), Algorithms for Euclidean neighbor relations, Boeing Document D180-17573-1, Boeing Aerospace Company, Seattle, Washington. 



\section{BIBLIOGRAPHIC DATA SHEET}

1. PULLCATION OR REPOAT MUMBEA NISTIR 4321

2. PERFOAMING ORGANIZATION REPORT NUMBER

3. PUEUCATION DATE

MAY 1990

\section{TITLE AND SUBTITLE}

On the Expected Complexity of the 3-Dimensional Voronoi Diagram

5. AUTHOR(S)

Javier Bernal

6. PERFOAMING ORGANIZATION (IF JOINT OR OTHER THAN NIST, SEE INSTAUCTIONS) U.S. DEPARTMENT OF COMMERCE

NATIONAL INSTITUTE OF STANDARDS AND TECHNOLOGY

GAITHERSBURG, MO 20899

7. CONTRACT/GRANT NUMBER

8. TYPE OF REPORT AND PERIOD COVERED

9. SPONSORING ORGANIZATION NAME AND COMPLETE ADDRESS (STREET, CITY, STATE, ZIP)

\section{SUPPLEMENTARY NOTES}

DOCUMENT DESCRIBES A COMPUTER PROGRAM; SF-185, FIPS SOFTWARE SUMMARY, IS ATTACHED.

11. ABSTAACT (A 200-WOAD OR LESS FACTUAL SUMMARY OF MOST SIONIFICANT INFORMATION. IF DOCUMENT INCLUDES A SIGNIFICANT BIBLOCRAPHY OR LTERATURE SURVEY, MENTION IT HERE.)

Let $\mathrm{S}$ be a set of $\mathrm{n}$ sites chosen independently from a uniform distribution in a cube in 3-dimensional Euclidean space. In this paper, work by Bentley, Weide and Yao is extended to show that the Voronoi diagram for $S$ has an expected $O(n)$ number of faces. A consequence of the proof of this result is that the Voronoi diagram for $S$ can be constructed in expected $O(n)$ time.

12. KEY WORDS (6 TO 12 ENTRIES; ALPHABETICAL OADER; CAPITALZE ONLY PROPER MAMES; AND SEPARATE KEY WORDS BY SEMICOLOMS) algorithm; computational geometry; expected complexity; expected linear; expected time analysis; Voronoi diagram

FOR OFFICIAL DISTRIBUTION. DO NOT RELEASE TO NATIONAL TECHNICAL INFORMATION SERVICE (NTIS).

ORDER FAOM SUPERINTENDENT OF DOCUMENTS, U.S. GOVERNMENT PRINTING OFFICE, WASHINGTON, DC 20402.

ORDEA FROM NATIONAL TECHNICAL INFORMATION SERVICE (NTIS), SPRINGFIELD, VA 22161.

14. NUMBER OF PAINTED PAGES

20

15. PRICE

$\mathrm{A} 02$ 


